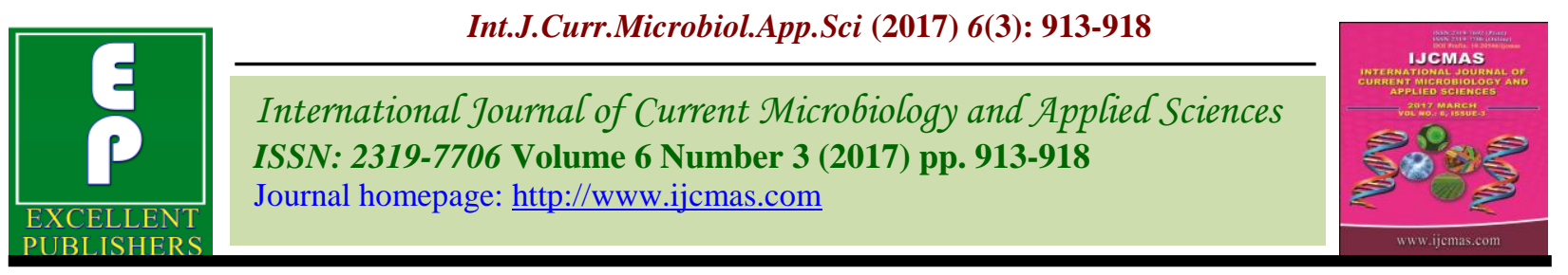

Original Research Article

https://doi.org/10.20546/ijcmas.2017.603.108

\title{
Study of Penicillin Resistance and Multidrug Resistance of Streptococcus pneumoniae in a Tertiary Care Hospital
}

\author{
L. Nandini*, G.B. Shantala and R. Ambika \\ ${ }^{1}$ Department of Microbiology, Bangalore Medical College and Research Institute, India \\ ${ }^{2}$ Department of Microbiology, BMCRI, India \\ *Corresponding author:
}

\begin{abstract}
A B S T R A C T
Streptococcus pneumoniae is the most common cause of community-acquired respiratory tract infections and one of the common causes of morbidity and mortality in developing countries. Penicillin has been the drug of choice for treatment of pneumococcal infections but there is increasing number of reports of penicillin resistant pneumococci (PRP)

\begin{tabular}{|c|}
\hline Keywords \\
\hline $\begin{array}{l}\text { Penicillin } \\
\text { Resistance, } \\
\text { Streptococcus } \\
\text { pneumoniae, } \\
\text { antibiotics }\end{array}$ \\
\hline Article Info \\
\hline $\begin{array}{l}\text { Accepted: } \\
\text { 18 February } 2017 \\
\text { Available Online: } \\
10 \text { March } 2017\end{array}$ \\
\hline
\end{tabular}
reported throughout the world. There is also an increase in multidrug-resistant strains. However, there are very less reports available from India. Aims: The aim of the study is to isolate and identify Streptococccus pneumoniae in clinical samples and to screen for penicillin resistance and study the antibiotic resistance pattern. S. pneumoniae was identified with Gram staining, bile solubility, inulin fermentation and sensitivity to optochin as per standard technique. Penicillin resistance was screened by oxacillin $(1 \mu \mathrm{g})$ disk on Muller-Hinton blood agar followed by Minimum Inhibitory Concentration (MIC) detection by the E-strip method according to the Clinical Laboratory Standards Institute (CLSI) guidelines. Antibiotic susceptibility for other antibiotics was carried out by the Kirby Bauer disk diffusion method followed by an E-test method. Out of 10 isolates, 20\% showed total resistance to penicillin, and $20 \%$ showed intermediate resistance. These penicillin-resistant pneumococci (20\%) were also found to be multidrug-resistant (MDR) strains. Maximum resistance was observed for cotrimoxazole $(60 \%)$, followed by tetracycline and cefotaxime (40\%). Conclusions: Increasing emergence of the resistant strains of $S$. pneumoniae in the community set up requires continuous monitoring and a restricted use of antibiotics to keep a check on its resistance pattern, for an effective treatment plan.
\end{abstract}

\section{Introduction}

Streptococcus pneumoniae is the major cause of community-acquired pneumonia. Commonly cause infection of the upper and lower respiratory tracts and are the most frequent bacterial cause of lobar and bronchopneumonia. They are also associated with acute bronchitis, acute exacerbation of chronic bronchitis, empyema, sinusitis, otitis media, meningitis, conjunctivitis, peritonitis, arthritis, and septicaemia (Winn et al., 2006).
Pneumococci are common inhabitants of the respiratory tract and may be isolated from the nasopharynx of 5-90\% of healthy persons, depending on the population and setting. Only $5-10 \%$ of adults without children are carriers. Among school-aged children, 20-60\% may be carriers. On military installations, as many as $50-60 \%$ of service personnel may be carriers. 
The duration of carriage varies and is generally longer in children than adults (Centers for Disease Control and Prevention, 2015). The World Health Organization (WHO) estimates that Streptococcus pneumoniae kills close to half a million children less than 5 years old worldwide every year, with most of these deaths occurring in developing countries.

Extremes of age or having some medical conditions can cause increased risk for pneumococcal disease. Children at increased risk for pneumococcal disease include those younger than 2 years old, in group child care, sickle cell disease, HIV infection, or chronic heart or lung conditions. Adults 65 years or older are at increased risk for pneumococcal disease, some adults 19 through 64 years old are also at increased risk for pneumococcal disease, including those, with chronic illnesses (lung, heart, liver, or kidney disease; asthma; diabetes; or alcoholism), with conditions that weaken the immune system (HIV/AIDS, cancer, or damaged/absent spleen), cochlear implants or cerebrospinal fluid (CSF) leaks (escape of the fluid that surrounds the brain and spinal cord).

A major concern for treatment of disease with Streptococcus pneumoniae is increasing frequency of antibiotic resistance, especially penicillin. Penicillin has been the drug of choice for treatment of pneumococcal infections but there is increasing number of reports of penicillin resistant pneumococci (PRP) reported throughout the world. There is also an increase in multidrug-resistant strains. This is of major concern as it can lead to treatment failure, prolonged hospital stay, thus increasing morbidity and mortality (Goyal et al., 2007; Chawla et al., 2010).

In India there are only few reports that show the resistance pattern in $S$. pneumoniae. With the increase in case fatality rates due to $S$. pneumoniae and worldwide emergence of drug resistance pneumococci it becomes necessary to do continuous surveillance of antimicrobial resistance patterns with emphasis on Penicillin Resistant Pneumococci (PRP). This study was undertaken to determine antibiotic susceptibility patterns and penicillin resistance rates of Streptococcus pneumoniae from the clinical samples isolated in a tertiary care center.

The main aim of this study is to isolate and identify Streptococccus pneumoniae in clinical samples and to study the antibiotic resistance pattern and to screen for penicillin resistance in clinical isolates of $S$. pneumoniae. Determine MIC for the antibiotics used.

\section{Materials and Methods}

The present prospective study was done in the Department of Microbiology, attached to Victoria Hospital, Bowring and Lady Curzon Hospital, Regional Institute of Ophthalmology and Vani Vilas Hospital, Bangalore Medical College and Research Institute, Bengaluru (BMCRI) from January 2015 to June 2015.

\section{Collection of bacterial isolates}

Streptococcus pneumoniae were isolated from various samples received in the laboratory. The samples were sputum (satisfied the Barlett grading), broncho alveolar lavage, nasopharyngeal swabs or aspirates, blood and cerebrospinal fluid. The following demographic data like age and sex of patient, diagnosis, sample, inpatient or outpatient and date of sample collection were recorded.

Culture of these samples was done on MacConkey agar and 5\% sheep blood agar which was incubated aerobically and anaerobically at $37^{\circ} \mathrm{C}$ for $18-24$ hours respectively. $S$. pneumoniae was suspected 
when there was partial clearing of blood and a greenish discolouration ( $\alpha$-haemolysis) produced underneath and in a narrow zone around the colonies on blood agar. The colonies on blood agar would be small, smooth and transparent, showing 'draughtsman form'. S. pneumoniae was identified with Gram staining, bile solubility, inulin fermentation and sensitivity to optochin. This identification was done as per standard technique (Ross, 2006).

\section{Antibiotic susceptibility testing}

Antibiotic susceptibility testing was done by Kirby Bauer disk diffusion method on Mueller-Hinton sheep blood agar. Oxacillin $(1 \mu \mathrm{g})$ disk was used for screening of Penicillin resistance. The other antibiotics tested were tetracycline $(30 \mu \mathrm{g})$, erythromycin $(15 \mu \mathrm{g})$, ciprofloxacin $(5 \mu \mathrm{g})$, cotrimoxazole $(25 \mu \mathrm{g})$, and cefotaxime $(30 \mu \mathrm{g})$. CLSI interpretive criteria were used to determine susceptibility.

\section{Minimum Inhibitory Concentration (MIC)}

Strains showing a zone diameter $\leq 19 \mathrm{~mm}$ around oxacillin $(1 \mu \mathrm{g})$ disc were tested for Minimum inhibitory concentrates (MICs) by Penicillin E-test strip. All other isolates which were resistant to other mentioned antibiotics according to CLSI guidance were tested by Etest strip. CLSI interpretive criteria were used to determine susceptibility.

\section{Results and Discussion}

Ten isolates of S.pneumoniae were obtained from clinical specimens (sputum, pleural fluid, blood, CSF, bronchoalveolar lavage, nasopharyngeal swabs and ear). Six were isolated from sputum, two from pleural fluid, one from cerebrospinal fluid and one from blood. Male to female ratio was 7:3 showing a male preponderance. Six isolates of $S$. pneumoniae were isolated from patients more than 50 years, three isolates from less than 5 years of age showing that S. pneumoniae is common in extremes of ages. Out of the ten patients, nine was inpatients and one was outpatient.

\section{Disc diffusion assay by Kirby Bauer disk diffusion method}

A total of 4 isolates (40\%) out of ten $S$. pneumoniae showed decreased susceptibility to penicillin by screening with oxacillin disc (1 $\mu \mathrm{g})$ diffusion test. Resistance to cotrimoxazole was detected in $60 \%$ of $S$. pneumoniae isolates. Resistance to tetracycline and cefotaxime was seen in $40 \%$.Resistance to erythromycin and ciprofloxacin was seen in $20 \%$ of the $S$. pneumoniae.

\section{Minimum inhibitory concentrationby E- strip method (Table 1)}

MIC of the 4(40\%) resistant strains by oxacillin screening test showed 2 (20\%) resistant and $2(20 \%)$ intermediate sensitivity by penicillin E-strip method. MIC by E-strip method for the 6 resistant cotrimoxazole showed 5 resistant and 1 intermediate sensitivity; 4 resistant tetracycline and cefotaxime as 3 resistant and 1 intermediate sensitivity, two erythromycin and ciprofloxacin resistant strains were resistant by disk diffusion and MIC by E-strip method.

In the present study maximum of $S$. pneumoniae were isolated from patients above 50 years suffering from lower respiratory tract infection. Preponderance of $S$. pneumoniae was more in the male patient about $70 \%$. S. pneumoniae infection are more common in extremes of ages (Breiman et al., 1994). Treatment of infections due to $S$. pneumoniae has become a complicated global problem due to antibiotic resistance. The 
emergence of resistance to penicillin in 1980's led to the increased use of macrolides, flouroquinolones and other drugs. While these antibiotics provided effective alternate therapy for $S$. pneumoniae infections, selective pressure associated with the widespread use of these antibiotics, has resulted in resistance to various classes of antimicrobial agents leading to multi-drug resistance.

In our study, $20 \%$ of the S. pneumoniae were resistant to penicillin, similar reports were made by several authors like Jyothikakshmi et al., (2015) from India 20.58\%; Trupl et al., (1997) from Slovak Republik 21\% and Vicioso from Spain 28\%. Other Indian studies reported were Goyal et al., (2007) 18\%, Ravi Kumar et al., (2014) 17\% and Upala Devi et al., (2012) 17\%. In contrast, a very low resistance rate of $1.5 \%$ was reported by the invasive bacterial infection surveillance group (IBIS) from India and other authors Reba $e t$ al., (2001) 7.3\%. Multidrug resistant $S$. pneumoniae, defined as resistant to penicillin and two or more non $\beta$ lactam agents such as macrolides, cotrimoxazole or tetracycline, are increasingly being reported from many parts of the globe (Lalitha et al., 2002). Penicillin susceptibility is an important marker for the presence or absence of a multidrug resistant phenotype. Strains with reduced susceptibility to penicillin are usually cross-resistant to other antibiotics. Moreover, it is also known that strain showing penicillin resistance can have genes responsible for resistance to other antibiotics. In our study, two (20\%) of isolates resistant to penicillin were multidrug resistant, one $(10 \%)$ isolate was resistant to cefotaxime and cotrimoxazole; and one (10\%) isolate was resistant to erythromycin; cefotaxime; ciprofloxacin and cotrimoxazole.

Table.1 Minimum inhibitory concentration by E-strip method

\begin{tabular}{|c|c|c|c|c|c|c|c|}
\hline Serial No. & Sample & $\begin{array}{l}\mathbf{P} \\
(\mu \mathrm{g} / \mathrm{mL})\end{array}$ & $\begin{array}{l}\mathbf{E} \\
(\mu \mathrm{g} / \mathrm{mL})\end{array}$ & $\begin{array}{l}\text { Ctx } \\
(\mu \mathrm{g} / \mathrm{mL})\end{array}$ & $\begin{array}{l}\text { Cip } \\
(\mu \mathrm{g} / \mathrm{mL})\end{array}$ & $\begin{array}{l}\text { Co-tri } \\
(\mu \mathrm{g} / \mathrm{mL})\end{array}$ & $\begin{array}{l}\text { T } \\
(\mu \mathrm{g} / \mathrm{mL})\end{array}$ \\
\hline 1. & $\begin{array}{l}\text { Pleural } \\
\text { Fluid }\end{array}$ & $\mathrm{IM}(4.2)$ & $\mathrm{S}(0.16)$ & $S(0.2)$ & $S(0.24)$ & $\mathrm{R}(4.0)$ & $\mathrm{R}(6.0)$ \\
\hline 2. & Sputum & S (1.8) & $\mathrm{S}(0.25)$ & $S(0.2)$ & $S(0.20)$ & $S(0.8)$ & S (1.0) \\
\hline 3. & Sputum & $\mathrm{R}(8.2)$ & $\mathrm{R}(1.8)$ & $\mathrm{R}(4.8)$ & $\mathrm{R}(20)$ & $\mathrm{R}(4.2)$ & S (0.8) \\
\hline 4. & Sputum & S (1.4) & $\mathrm{S}(0.20)$ & S (0.4) & $\mathrm{S}(0.22)$ & S (0.6) & S (1.0) \\
\hline 5. & CSF & $\mathrm{R}(0.14)$ & $\mathrm{S}(0.14)$ & $\mathrm{R}(6.0)$ & $\mathrm{S}(0.10)$ & $\mathrm{R}(4.0)$ & S (0.6) \\
\hline 6. & $\begin{array}{l}\text { Pleural } \\
\text { fluid }\end{array}$ & S (1.8) & $\mathrm{S}(0.22)$ & $\mathrm{R}(4.0)$ & $\mathrm{R}(10)$ & R (4.4) & $\mathrm{R}(4.2)$ \\
\hline 7. & Sputum & S (1.4) & $\mathrm{R}(1.2)$ & $\operatorname{IM}(2.2)$ & S (0.24) & S (0.6) & IM (3.2) \\
\hline 8. & Sputum & S (1.6) & $\mathrm{S}(0.25)$ & S (1.0) & $\mathrm{S}(0.20)$ & S (0.6) & $\mathrm{R}(4.8)$ \\
\hline 9. & Sputum & IM (4.0) & $\mathrm{S}(0.12)$ & $S(0.2)$ & $\mathrm{S}(0.24)$ & IM (2) & S (0.8) \\
\hline 10. & Blood & S (1.6) & $\mathrm{S}(0.24)$ & $S(0.4)$ & $\mathrm{S}(0.20)$ & $\mathrm{R}(4.0)$ & $\mathrm{S}(0.4)$ \\
\hline
\end{tabular}

$\mathrm{P}=$ Penicillin $; \mathrm{E}=$ Erythromycin $; \mathrm{Ctx}=$ Cefotaxime Cip = Ciprofloxacin Co-tri $=$ Cotrimoxazole $; \mathrm{T}=$ Tetracycline; $\mathrm{R}=$ Resistant; IM = Intermediate resistant; $\mathrm{S}=$ Sensitive. 
Table. 2 Comparison of penicillin resistance among various studies

\begin{tabular}{|l|c|}
\hline Study & Penicillin Resistance \\
\hline Present Study (2015) & $20 \%$ \\
\hline Jyothilakshmi (2015) & $20.5 \%$ \\
\hline Ravi Kumar et al., (2014) & $17 \%$ \\
\hline Upala Devi et al., (2012) & $17 \%$ \\
\hline Chawla et al., (2010) & $14 \%$ \\
\hline Goyal et al., (2007) & $18.3 \%$ \\
\hline Reba et al., (2001) & $7.3 \%$ \\
\hline IBIS (1999) & $1.5 \%$ \\
\hline
\end{tabular}

The increased rate of resistance to these antibiotics can be possibly correlated with the wide use of these antibiotics in communities, because of their dose convenience, cost effectiveness, and also, their easy availability over the counter (Rijal et al., 2010). This is a matter of great concern, as it results in higher morbidity, mortality. May also lead to higher rate of treatment failure, additional cost of therapy and extra days of hospitalization, the increasing penicillin and multidrug resistance of S. pneumoniae worldwide has important clinical implications. Routine screening for antibiotic susceptibility, judicious use of antibiotics, continued surveillance and attention to pneumococcal vaccination is advocated to inhibit the constant increase in resistance in pneumococci.

In conclusion, indiscriminate use of antibiotics at an inappropriate dosage at the community level might be the probable cause of resistance. Therefore, there should be a restraint for the indiscrete use of antibiotics, to limit the surfacing of resistant strains. Emergence of resistant strains and also the MDR strains of $S$. pneumoniae need continuous local as well as global monitoring of the sensitivity pattern, so as to plan the line of treatment.

Limitations of the study: This study was done for a relatively short duration for small number of samples. It may not reflect the national status of pathogen distribution or the antimicrobial resistance pattern.

\section{Acknowledgement}

We thank the institute Bangalore Medical College and Research Institute to conduct this study.

\section{References}

Breiman, R.F., et al. 1994. Emergence of drug-resistant pneumococcal infections in the United States. JAMA, 271: 18315.

Centers for Disease Control and Prevention. 2015. Streptococcus pneumonia. National Center and Respiratory Diseases, Division of Bacterial Diseases.

Chawla, K., et al. 2010. Reporting Emerging Resistance of Streptococcus pneumonia from India. J. Glob. Infect. Dis., 2(1): $10-14$.

Clinical and Laboratory Standards Institute. 2014. M100-S24. Performance Standards for Antimicrobial Susceptibility Testing; Twenty Fourth Informational Supplement, 88-93.

Goyal, R., et al. 2007. Antimicrobial resistance in invasive and colonizing Streptococcus pneumonia in North India. Indian J. Med. Microbiol., 25: 256-9. 
Invasive Bacterial infection surveillance (IBIS) group. 1999. Prospective multicentre hospital surveillance of Streptococcus pneumoniae disease in India. Lancet, 353(9160): 1216-21.

Jyothilakshmi, et al. 2015. Emerging Resistance and Antibiotic Susceptibility Patterns in Streptococcus pneumoniae. Int. J. Curr. Med. Appl. Sci., 7(1): 3033.

Kanungo, R., et al. 2001. Serotype distribution and antimicrobial resistance in Streptococcus pneumoniae causing invasive and other infections in South India. Indian J. Med. Res., 114: 127-32.

Lalitha, M.K., et al. 2002. Multidrug-resistant Streptococcus pneumoniae from India. The Lancet, 3: 445.

Ravi Kumar, K.L., et al. 2014. Nasopharyngeal carriage, antibiogram and serotype distribution of Streptococcus pneumoniae among healthy under five children. Indian $J$. Med. Res., 140: 216-220.

Rijal, B., et al. 2010. Antimicrobial susceptibility pattern and serotyping of Streptococcus pneumoniae isolated from Kanti Children Hospital in Nepal.
Kathmandu Univ. Med. J., 8(30): 1648.

Ross, P.W. 2006. "Streptococci and Enterococci" Mackie and Mccartney's Practical Medical Microbiology, 14th ed Elsievier, 268-269.

Trupl, J., et al. 1997.. The incidence of penicillin - resistant pneumococci in the Slovak Republik - Pneumococcus study group. Chemother., 43(5): 316 - 22.

Utpala Devi, et al. 2012. Serotype distribution and sensitivity pattern of nasopharyngeal colonizing Streptococcus pneumoniae among rural children of eastern India. Indian J. Med. Res., 136: 495-498.

Vicioso, D., et al. 1991. Serotype distribution and antimicrobial resistance of Streptococcus pneumoniaeisolates causing systemic infection in Spain 1979 -1989. Rev. infect. Dis., 13(1): 5660.

Winn, Washington, C., Koneman, Elmer, W. 2006. Streptococcus pneumonia. Koneman's Color Atlas And Textbook of Diagnostic Microbiology. $6^{\text {th }}$ ed. Philadelphia: Lippincott Williams and Wilkins, 275-281.

\section{How to cite this article:}

Nandini, L., G.B. Shantala and Ambika, R. 2017. Study of Penicillin Resistance and Multidrug Resistance of Steptococcus pneumoniae in a Tertiary Care Hospital. Int.J.Curr.Microbiol.App.Sci. 6(3): 913-918. doi: https://doi.org/10.20546/ijcmas.2017.603.108 